Acta Crystallographica Section E

Structure Reports

Online

ISSN 1600-5368

\section{(2Z,3Z)-Quinoxaline-2,3(1H,4H)-dione dioxime}

\section{Ali Kakanejadifard ${ }^{a *}$ and Vahid Amani ${ }^{b}$}

aDepartment of Chemistry, Faculty of Science, Lorestan University, Khorramabad, Iran, and ${ }^{\mathbf{b}}$ Department of Chemistry, Islamic Azad University, Shahr-e-Rey Branch, Tehran, Iran

Correspondence e-mail: alikakanejadifard@yahoo.com

Received 11 July 2008; accepted 11 July 2008

Key indicators: single-crystal X-ray study; $T=120 \mathrm{~K}$; mean $\sigma(\mathrm{C}-\mathrm{C})=0.003 \AA$; $R$ factor $=0.060 ; w R$ factor $=0.118 ;$ data-to-parameter ratio $=14.7$.

The asymmetric unit of the title compound, $\mathrm{C}_{8} \mathrm{H}_{8} \mathrm{~N}_{4} \mathrm{O}_{2}$, contains one half-molecule; a twofold rotation axis bisects the molecule. An intramolecular $\mathrm{N}-\mathrm{H}$...O hydrogen bond results in the formation of a five-membered ring, which displays an envelope conformation. In the crystal structure, intermolecular $\mathrm{O}-\mathrm{H} \cdots \mathrm{N}$ hydrogen bonds link the molecules.

\section{Related literature}

For related literature, see: Kakanejadifard, Niknam \& Zabardasti (2007); Kakanejadifard, Saniei et al. (2007); Kakanejadifard \& Niknam (2006); For general background, see: Jones et al. (1961); Schrauzer \& Kohnle (1964); Yari et al. (2006); Hashemi et al. (2006); Ghiasvand et al. (2004, 2005); Kakanejadifard, Niknam, Ranjbar et al. (2007); Gok \& Kantekin (1997).<smiles></smiles>

\section{Experimental}

Crystal data

$\mathrm{C}_{8} \mathrm{H}_{8} \mathrm{~N}_{4} \mathrm{O}_{2}$

$M_{r}=192.18$

Orthorhombic, Fddd

$a=9.831$ (2) §

$b=13.609(3) \AA$

$c=24.344(5) \AA$
Data collection

Bruker SMART 1000 CCD area-detector diffractometer Absorption correction: none 7925 measured reflections

independent reflections 708 reflections with $I>2 \sigma(I)$ $R_{\text {int }}=0.065$

Refinement

$R\left[F^{2}>2 \sigma\left(F^{2}\right)\right]=0.060$

$w R\left(F^{2}\right)=0.118$

$S=1.01$

983 reflections

67 parameters

1 restraint

$\mathrm{H}$ atoms treated by a mixture of independent and constrained refinement $\Delta \rho_{\max }=0.43{\mathrm{e} \AA^{-3}}^{-3}$ $\Delta \rho_{\min }=-0.25 \mathrm{e}^{-3}$

Table 1

Hydrogen-bond geometry $\left(\AA{ }^{\circ}\right)$.

\begin{tabular}{lllll}
\hline$D-\mathrm{H} \cdots A$ & $D-\mathrm{H}$ & $\mathrm{H} \cdots A$ & $D \cdots A$ & $D-\mathrm{H} \cdots A$ \\
\hline $\mathrm{O} 1-\mathrm{H} 1 \cdots \mathrm{N} 1^{\mathrm{i}}$ & $0.852(13)$ & $1.971(10)$ & $2.763(2)$ & 154 \\
$\mathrm{~N} 2-\mathrm{H} 2 A \cdots \mathrm{O} 1$ & 0.86 & 2.25 & $2.566(3)$ & 102
\end{tabular}

Symmetry code: (i) $-x+1,-y,-z+1$.

Data collection: SMART (Bruker, 1998); cell refinement: SMART; data reduction: SAINT-Plus (Bruker, 1998); program(s) used to solve structure: SHELXTL (Sheldrick, 2008); program(s) used to refine structure: SHELXTL; molecular graphics: SHELXTL; software used to prepare material for publication: $S H E L X T L$.

The authors are grateful to the Research Grant Council of Lorestan University for financial support.

Supplementary data and figures for this paper are available from the IUCr electronic archives (Reference: HK2493).

\section{References}

Bruker (1998). SAINT-Plus and SMART. Bruker AXS Inc., Madison, Wisconsin, USA.

Ghiasvand, A. R., Ghaderi, R. \& Kakanejadifard, A. (2004). Talanta, 62, $287-$ 292.

Ghiasvand, A. R., Shadabi, S., Kakanejadifard, A. \& Khajehkolaki, A. (2005). Bull. Korean Chem. Soc. 26, 781-785.

Gok, Y. \& Kantekin, H. (1997). Polyhedron, 16, 2413-2420.

Hashemi, P., Rahmani, Z., Kakanejadifard, A. \& Niknam, E. (2006). Anal. Sci. 21, 1297-1301

Jones, M. E. B., Thornton, D. A. \& Webb, R. F. (1961). Makromol. Chem. 49, $62-66$.

Kakanejadifard, A. \& Niknam, E. (2006). Pol. J. Chem. 80, 1645-1649.

Kakanejadifard, A., Niknam, E., Ranjbar, B. \& Naderi-Manesh, H. (2007). Synth. Commun. 37, 2753-2756.

Kakanejadifard, A., Niknam, E. \& Zabardasti, A. (2007). J. Coord. Chem. 60, $677-681$.

Kakanejadifard, A., Saniei, A., Delfani, F., Farnia, M. \& Najafi, G. R. (2007). J. Heterocycl. Chem. 44, 717-718.

Schrauzer, G. N. \& Kohnle, J. (1964). Chem. Ber. 97, 3056-3063.

Sheldrick, G. M. (2008). Acta Cryst. A64, 112-122.

Yari, A., Azizi, S. \& Kakanejadifard, A. (2006). Sens. Actuators B, 119, 167173. 


\section{supporting information}

Acta Cryst. (2008). E64, o1512 [doi:10.1107/S1600536808021570]

\section{(2Z,3Z)-Quinoxaline-2,3(1H,4H)-dione dioxime}

\section{Ali Kakanejadifard and Vahid Amani}

\section{S1. Comment}

Recently, we have reported the syntheses and chemical behaviours of some vic-dioximes. In our investigations, the reactions of amines with dichloro- glyoxime or cyanogendi-N-oxide resulted in various symmetrically substituted diaminoglyoxime derivatives, in which some of them were quite suitable to act, as donor species, towards some transition metal ions (Kakanejadifard, Niknam \& Zabardasti, 2007; Kakanejadifard, Saniei et al., 2007; Kakanejadifard \& Niknam, 2006). Some oximes are widely used for various purposes in organic, inorganic, bioinorganic, pigment, analytical, dyes and medical chemistry (Jones et al., 1961; Schrauzer \& Kohnle, 1964; Yari et al., 2006; Hashemi et al., 2006; Ghiasvand et al., 2004, 2005; Kakanejadifard, Niknam, Ranjbar et al., 2007). vic-Dioximes, containing mildly acidic hydroxyl groups and slightly basic nitrogen atoms, are amphoteric and their transition metal complexes have been widely investigated as analytical reagents (Gok \& Kantekin, 1997). We report herein the synthesis and crystal structure of the title compound.

The asymmetric unit of the title compound contains one-half molecule (Fig. 1). The intramolecular $\mathrm{N}-\mathrm{H} \cdots \mathrm{O}$ hydrogen bond (Table 1) results in the formation of a five-membered ring: (O1/N1/N2/C1/H2A), having envelope conformation, with $\mathrm{H} 2 \mathrm{~A}$ atom displaced by $-0.132 \AA$ from the plane of the other ring atoms.

In the crystal structure, intermolecular O-H $\cdots \mathrm{N}$ hydrogen bonds (Table 1) link the molecules (Fig. 2), in which they may be effective in the stabilization of the structure.

\section{S2. Experimental}

For the preparation of the title compound, a solution of $\mathrm{Na}_{2} \mathrm{CO}_{3}(0.2 \mathrm{~g}, 1.9 \mathrm{mmol})$ in $\mathrm{MeCN}(30 \mathrm{ml})$ was added to a magnetically stirred solution of dicholoroglyoxime $(1.57 \mathrm{~g}, 10 \mathrm{mmol})$ in $\mathrm{MeCN}(20 \mathrm{ml})$ and a solution of 1,2-phenylendiamine $(1.08 \mathrm{~g}, 10 \mathrm{mmol})$ at $278 \mathrm{~K}$. After $2 \mathrm{~h}$ stirring at room temperature, the mixture was filtered and the brown precipitate was washed with cold MeCN. It was recrystallized from $\mathrm{EtOH}(70 \%$ aq) in one week (yield; 93.0\%, m.p. 512 $\mathrm{K})$.

\section{S3. Refinement}

$\mathrm{H} 1$ atom (for $\mathrm{OH}$ ) was located in difference syntheses and refined [O- $\mathrm{H}=0.852$ (13) $\left.\AA, \mathrm{U}_{\text {iso }}(\mathrm{H})=0.039 \AA^{2}\right]$. The remaining $\mathrm{H}$ atoms were positioned geometrically, with $\mathrm{N}-\mathrm{H}=0.86 \AA$ (for $\mathrm{NH}$ ) and $\mathrm{C}-\mathrm{H}=0.93 \AA$ for aromatic $\mathrm{H}$, and constrained to ride on their parent atoms with $\mathrm{U}_{\text {iso }}(\mathrm{H})=1.2 \mathrm{U}_{\mathrm{eq}}(\mathrm{C}, \mathrm{N})$. 


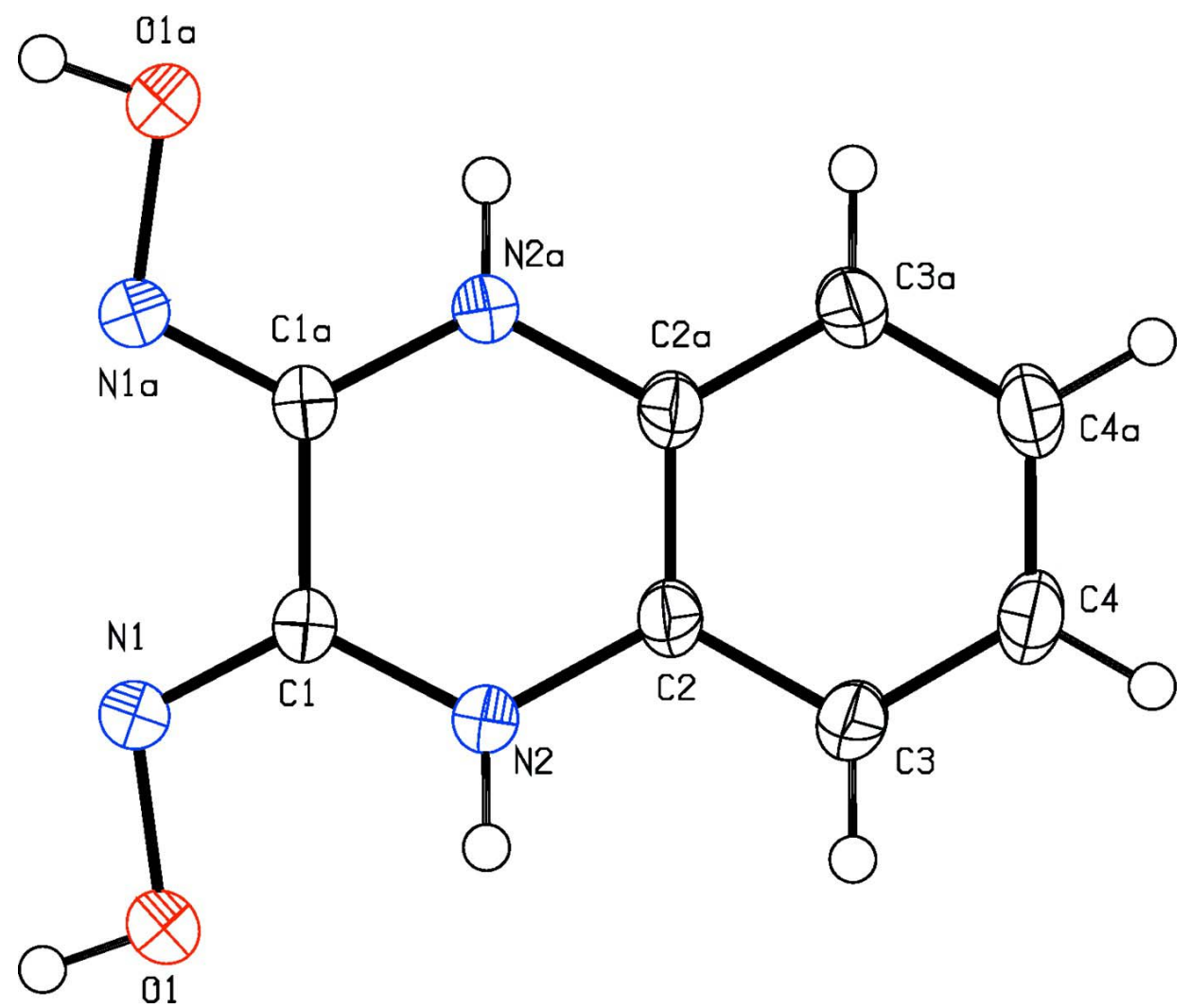

Figure 1

The molecular structure of the title molecule, with the atom-numbering scheme. Displacement ellipsoids are drawn at the $50 \%$ probability level [symmetry code: (a) $5 / 4-\mathrm{x}, 1 / 4-\mathrm{y}, \mathrm{z}$ ]. 


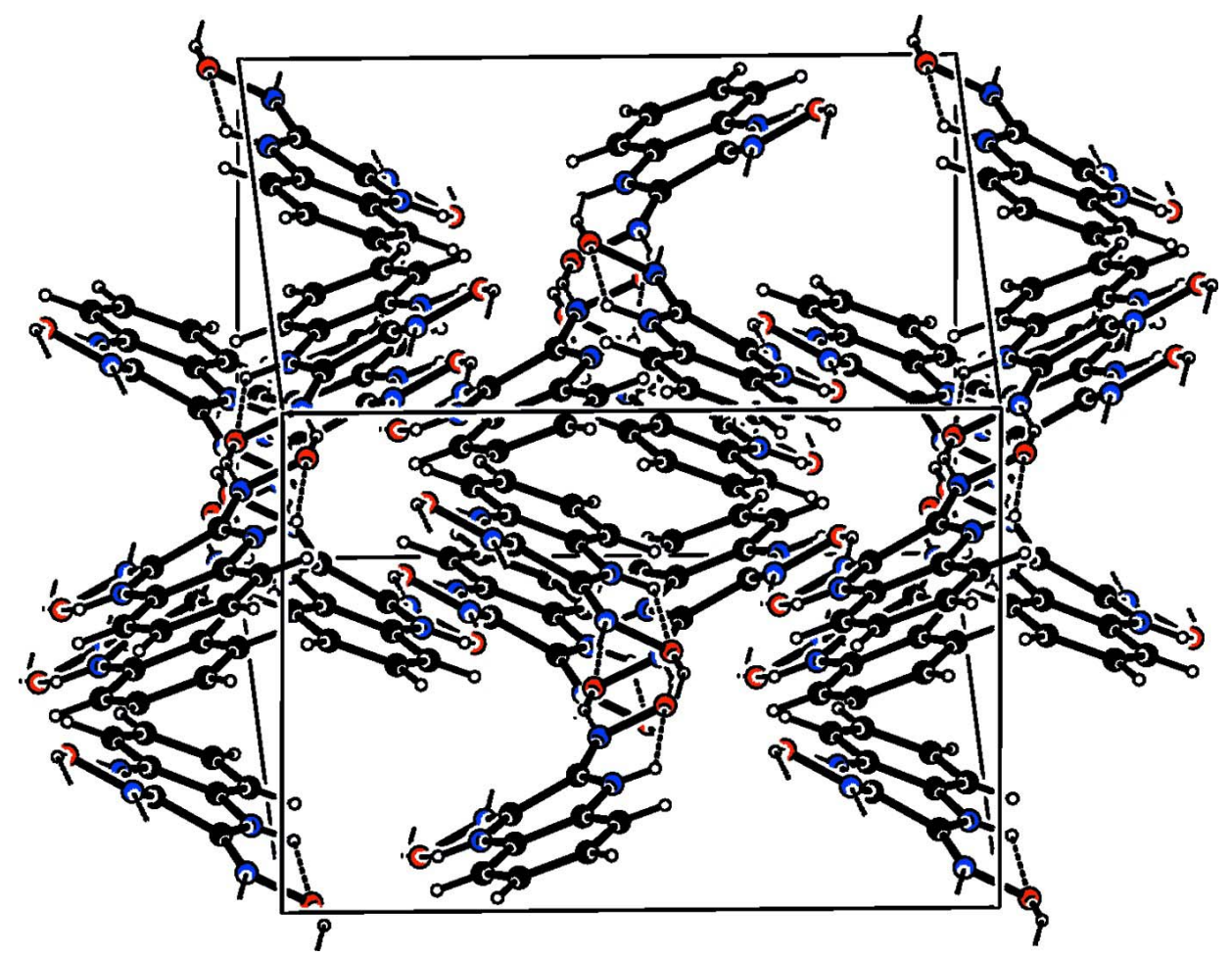

Figure 2

A packing diagram of the title molecule. Hydrogen bonds are shown as dashed lines.

(2Z,3Z)-Quinoxaline-2,3(1H,4H)-dione dioxime

Crystal data

$\mathrm{C}_{8} \mathrm{H}_{8} \mathrm{~N}_{4} \mathrm{O}_{2}$

$M_{r}=192.18$

Orthorhombic, Fddd

Hall symbol: -F $2 \mathrm{uv} 2 \mathrm{vw}$

$a=9.831(2) \AA$

$b=13.609(3) \AA$

$c=24.344(5) \AA$

$V=3256.9(11) \AA^{3}$

$Z=16$

\section{Data collection}

Bruker SMART 1000 CCD area-detector diffractometer

Radiation source: fine-focus sealed tube

Graphite monochromator

$\varphi$ and $\omega$ scans

7925 measured reflections

983 independent reflections
$F(000)=1600$

$D_{\mathrm{x}}=1.568 \mathrm{Mg} \mathrm{m}^{-3}$

Mo $K \alpha$ radiation, $\lambda=0.71073 \AA$

Cell parameters from 744 reflections

$\theta=3-30^{\circ}$

$\mu=0.12 \mathrm{~mm}^{-1}$

$T=120 \mathrm{~K}$

Prism, light-brown

$0.4 \times 0.2 \times 0.2 \mathrm{~mm}$

708 reflections with $I>2 \sigma(I)$

$R_{\text {int }}=0.065$

$\theta_{\max }=28.0^{\circ}, \theta_{\min }=2.7^{\circ}$

$h=-12 \rightarrow 12$

$k=-17 \rightarrow 17$

$l=-32 \rightarrow 31$ 


\section{Refinement}

Refinement on $F^{2}$

Least-squares matrix: full

$R\left[F^{2}>2 \sigma\left(F^{2}\right)\right]=0.060$

$w R\left(F^{2}\right)=0.118$

$S=1.01$

983 reflections

67 parameters

1 restraint

Primary atom site location: structure-invariant direct methods
Secondary atom site location: difference Fourier map

Hydrogen site location: difference Fourier map

$\mathrm{H}$ atoms treated by a mixture of independent and constrained refinement

$w=1 /\left[\sigma^{2}\left(F_{\mathrm{o}}^{2}\right)+(0.005 P)^{2}+25 P\right]$ where $P=\left(F_{\mathrm{o}}^{2}+2 F_{\mathrm{c}}^{2}\right) / 3$

$(\Delta / \sigma)_{\max }<0.001$

$\Delta \rho_{\max }=0.43{\mathrm{e} \AA^{-3}}^{-3}$

$\Delta \rho_{\min }=-0.25$ e $\AA^{-3}$

\section{Special details}

Geometry. All e.s.d.'s (except the e.s.d. in the dihedral angle between two 1.s. planes) are estimated using the full covariance matrix. The cell e.s.d.'s are taken into account individually in the estimation of e.s.d.'s in distances, angles and torsion angles; correlations between e.s.d.'s in cell parameters are only used when they are defined by crystal symmetry. An approximate (isotropic) treatment of cell e.s.d.'s is used for estimating e.s.d.'s involving l.s. planes.

Refinement. Refinement of $\mathrm{F}^{2}$ against ALL reflections. The weighted R-factor $\mathrm{wR}$ and goodness of fit $\mathrm{S}$ are based on $\mathrm{F}^{2}$, conventional $R$-factors $R$ are based on $F$, with $F$ set to zero for negative $F^{2}$. The threshold expression of $F^{2}>2$ sigma $\left(F^{2}\right)$ is used only for calculating R-factors(gt) etc. and is not relevant to the choice of reflections for refinement. R-factors based on $\mathrm{F}^{2}$ are statistically about twice as large as those based on F, and R- factors based on ALL data will be even larger.

Fractional atomic coordinates and isotropic or equivalent isotropic displacement parameters $\left(\AA^{2}\right)$

\begin{tabular}{lllll}
\hline & $x$ & $y$ & $z$ & $U_{\text {iso }} / U_{\text {eq }}$ \\
\hline O1 & $0.47468(17)$ & $-0.04564(12)$ & $0.43944(6)$ & $0.0324(4)$ \\
$\mathrm{H} 1$ & $0.454(3)$ & $-0.0611(18)$ & $0.4723(4)$ & $0.039^{*}$ \\
$\mathrm{~N} 1$ & $0.54273(19)$ & $0.04548(13)$ & $0.44726(7)$ & $0.0261(4)$ \\
$\mathrm{N} 2$ & $0.5691(2)$ & $0.03307(14)$ & $0.35156(7)$ & $0.0308(5)$ \\
$\mathrm{H} 2 \mathrm{~A}$ & 0.5385 & -0.0261 & 0.3513 & $0.037^{*}$ \\
$\mathrm{C} 1$ & $0.5867(2)$ & $0.07818(15)$ & $0.40084(8)$ & $0.0242(4)$ \\
$\mathrm{C} 2$ & $0.5981(2)$ & $0.07776(16)$ & $0.30119(8)$ & $0.0268(5)$ \\
$\mathrm{C} 3$ & $0.5745(2)$ & $0.03025(19)$ & $0.25164(9)$ & $0.0358(6)$ \\
$\mathrm{H} 3 \mathrm{~A}$ & 0.5413 & -0.0337 & 0.2514 & $0.043^{*}$ \\
$\mathrm{C} 4$ & $0.6000(3)$ & $0.0776(2)$ & $0.20291(9)$ & $0.0412(6)$ \\
$\mathrm{H} 4 \mathrm{~A}$ & 0.5835 & 0.0457 & 0.1698 & $0.049^{*}$ \\
\hline
\end{tabular}

Atomic displacement parameters $\left(\AA^{2}\right)$

\begin{tabular}{lllllll}
\hline & $U^{11}$ & $U^{22}$ & $U^{33}$ & $U^{12}$ & $U^{13}$ & $U^{23}$ \\
\hline $\mathrm{O} 1$ & $0.0423(10)$ & $0.0304(8)$ & $0.0244(7)$ & $-0.0098(7)$ & $0.0009(7)$ & $0.0025(7)$ \\
$\mathrm{N} 1$ & $0.0314(9)$ & $0.0243(9)$ & $0.0226(8)$ & $-0.0053(8)$ & $0.0002(7)$ & $0.0004(7)$ \\
$\mathrm{N} 2$ & $0.0447(11)$ & $0.0279(9)$ & $0.0198(8)$ & $-0.0126(8)$ & $0.0022(8)$ & $-0.0015(7)$ \\
$\mathrm{C} 1$ & $0.0282(11)$ & $0.0261(10)$ & $0.0184(9)$ & $-0.0014(9)$ & $-0.0009(8)$ & $-0.0002(8)$ \\
$\mathrm{C} 2$ & $0.0282(11)$ & $0.0334(11)$ & $0.0187(9)$ & $-0.0055(9)$ & $0.0016(8)$ & $-0.0002(8)$ \\
$\mathrm{C} 3$ & $0.0422(13)$ & $0.0422(13)$ & $0.0230(10)$ & $-0.0154(11)$ & $0.0041(10)$ & $-0.0059(10)$ \\
$\mathrm{C} 4$ & $0.0440(14)$ & $0.0596(17)$ & $0.0199(10)$ & $-0.0188(13)$ & $0.0004(10)$ & $-0.0054(10)$ \\
\hline
\end{tabular}


Geometric parameters $\left(\AA,{ }^{\circ}\right)$

\begin{tabular}{llll}
\hline $\mathrm{O} 1-\mathrm{H} 1$ & $0.852(13)$ & $\mathrm{C} 2-\mathrm{C} 2^{\mathrm{i}}$ & $1.390(4)$ \\
$\mathrm{N} 1-\mathrm{C} 1$ & $1.289(3)$ & $\mathrm{C} 2-\mathrm{C} 3$ & $1.388(3)$ \\
$\mathrm{N} 1-\mathrm{O} 1$ & $1.422(2)$ & $\mathrm{C} 3-\mathrm{C} 4$ & $1.373(3)$ \\
$\mathrm{N} 2-\mathrm{C} 1$ & $1.359(2)$ & $\mathrm{C} 3-\mathrm{H} 3 \mathrm{~A}$ & 0.9300 \\
$\mathrm{~N} 2-\mathrm{C} 2$ & $1.398(3)$ & $\mathrm{C} 4-\mathrm{C} 4^{\mathrm{i}}$ & 0.9300 \\
$\mathrm{~N} 2-\mathrm{H} 2 \mathrm{~A}$ & 0.8600 & $\mathrm{C} 4-\mathrm{H} 4 \mathrm{~A}$ & \\
$\mathrm{C} 1-\mathrm{C} 1^{\mathrm{i}}$ & $1.481(4)$ & & $121.7(2)$ \\
& & & $118.68(11)$ \\
$\mathrm{N} 1-\mathrm{O} 1-\mathrm{H} 1$ & $101.8(17)$ & $\mathrm{C} 3-\mathrm{C} 2-\mathrm{N} 2$ & $120.1(2)$ \\
$\mathrm{C} 1-\mathrm{N} 1-\mathrm{O} 1$ & $109.96(16)$ & $\mathrm{C} 2-\mathrm{C} 2-\mathrm{N} 2$ & 120.0 \\
$\mathrm{C} 1-\mathrm{N} 2-\mathrm{C} 2$ & $123.50(18)$ & $\mathrm{C} 4-\mathrm{C} 3-\mathrm{C} 2$ & 120.0 \\
$\mathrm{C} 1-\mathrm{N} 2-\mathrm{H} 2 \mathrm{~A}$ & 118.3 & $\mathrm{C} 4-\mathrm{C} 3-\mathrm{H} 3 \mathrm{~A}$ & $120.25(14)$ \\
$\mathrm{C} 2-\mathrm{N} 2-\mathrm{H} 2 \mathrm{~A}$ & 118.3 & $\mathrm{C} 2-\mathrm{C} 3-\mathrm{H} 3 \mathrm{~A}$ & 119.9 \\
$\mathrm{~N} 1-\mathrm{C} 1-\mathrm{N} 2$ & $125.13(19)$ & $\mathrm{C} 3-\mathrm{C} 4-\mathrm{C} 4$ & 119.9 \\
$\mathrm{~N} 1-\mathrm{C} 1-\mathrm{C} 1^{\mathrm{i}}$ & $117.89(12)$ & $\mathrm{C} 3-\mathrm{C} 4-\mathrm{H} 4 \mathrm{~A}$ & \\
$\mathrm{~N} 2-\mathrm{C} 1-\mathrm{C} 1^{\mathrm{i}}$ & $116.98(12)$ & $\mathrm{C} 4-\mathrm{C} 4-\mathrm{H} 4 \mathrm{~A}$ & $-11.4(4)$ \\
$\mathrm{C} 3-\mathrm{C} 2-\mathrm{C} 2^{\mathrm{i}}$ & $119.62(13)$ & & $-2.1(4)$ \\
& & & $177.9(2)$ \\
$\mathrm{C} 1-\mathrm{N} 2-\mathrm{C} 2-\mathrm{C} 3$ & $-177.8(2)$ & $\mathrm{C} 2-\mathrm{N} 2-\mathrm{C} 1-\mathrm{C} 1^{\mathrm{i}}$ & $0.4(5)$ \\
$\mathrm{C} 1-\mathrm{N} 2-\mathrm{C} 2-\mathrm{C} 2^{\mathrm{i}}$ & $2.2(4)$ & $\mathrm{C} 2-\mathrm{C} 2-\mathrm{C} 3-\mathrm{C} 4$ & \\
$\mathrm{O} 1-\mathrm{N} 1-\mathrm{C} 1-\mathrm{N} 2$ & $0.8(3)$ & $\mathrm{N} 2-\mathrm{C} 2-\mathrm{C} 3-\mathrm{C} 4$ & \\
$\mathrm{O} 1-\mathrm{N} 1-\mathrm{C} 1-\mathrm{C} 1^{\mathrm{i}}$ & $-178.7(2)$ & $\mathrm{C} 2-\mathrm{C} 3-\mathrm{C} 4-\mathrm{C} 4{ }^{\mathrm{i}}$ & \\
$\mathrm{C} 2-\mathrm{N} 2-\mathrm{C} 1-\mathrm{N} 1$ & $169.1(2)$ & & \\
& & & \\
\hline
\end{tabular}

Symmetry code: (i) $-x+5 / 4,-y+1 / 4, z$.

Hydrogen-bond geometry $\left(A,{ }^{\circ}\right)$

\begin{tabular}{lllll}
\hline$D-\mathrm{H}^{\cdots} A$ & $D-\mathrm{H}$ & $\mathrm{H} \cdots A$ & $D \cdots A$ & $D-\mathrm{H} \cdots A$ \\
\hline $\mathrm{O} 1-\mathrm{H} 1 \cdots \mathrm{N} 1^{\mathrm{ii}}$ & $0.85(1)$ & $1.97(1)$ & $2.763(2)$ & 154 \\
$\mathrm{~N} 2-\mathrm{H} 2 A \cdots \mathrm{O} 1$ & 0.86 & 2.25 & $2.566(3)$ & 102 \\
\hline
\end{tabular}

Symmetry code: (ii) $-x+1,-y,-z+1$. 